\title{
Billion Organisms
}

National Cancer Institute

\section{Source}

National Cancer Institute. Billion Organisms. NCI Thesaurus. Code C71189.

A unit of measure of quantity of organisms expressed in billions. 